\title{
The Influence of Film Thickness on the Microstructure of Nanocrystalline Nickel Films: A Precession Electron Diffraction Microscopy Study
}

\author{
S. Rajasekhara,* K. J. Ganesh,* K. Hattar**, J. A. Knapp**, P. J. Ferreira,* \\ * Materials Science \& Engineering Program, The University of Texas - Austin, Austin, TX \\ 78712 \\ ** Sandia National Laboratories, Albuquerque, NM 87185
}

Recent studies have indicated that pulsed laser deposition (PLD) nanocrystalline nickel films exhibit special properties relative to PLD nanocrystalline copper or gold films [1]. In particular, in-situ TEM heating experiments have demonstrated the presence of large $h c p$ phase nickel grains $(>100 \mathrm{~nm})$ within a $f c c$ phase nanocrystalline nickel matrix $[2,3]$. However, it is currently unknown whether nanoscale $h c p$ nickel grains are present in as-deposited films due to the limited spatial resolution of conventional characterization techniques for phase mapping. To address this issue, a Diffraction-Scanning Transmission Electron Microscopy (D-STEM) type lens configuration [4] combined with precession electron microscopy [5], capable of spatial resolution $<10 \mathrm{~nm}$, has been used to determine the distribution of $f c c$ and $h c p$ nickel phases, as well as the grain size distribution of nanocrystalline nickel films of different thicknesses (50 and $100 \mathrm{~nm})$.

Bright-field TEM images from the 50 and $100 \mathrm{~nm}$-thick PLD nickel films are shown in Figs. 1a and $1 \mathrm{~b}$, respectively. Although several nanoscale grains are visible in these images, dynamical electron scattering, coupled with the fact that some grains are oriented off-zone axis, severely limits the overall microstructure analysis. In contrast, the reconstructed image containing phase and reliablity information obtained by D-STEM and precession electron microscopy clearly shows, for the first time, the true morphology of the nanocrystalline PLD nickel film (Figs. 1c and 1d). Both $h c p$ and $f c c$ phase grains with distinct grain boundaries are visible in the $50 \mathrm{~nm}$ film, while in the case of the $100 \mathrm{~nm}$-thick film distinct $h c p$ nickel grains appear to be located at the $f c c$ grain boundaries. A careful analysis of approximately 831 and $956 h c p$ phase grains in the 50 and the $100 \mathrm{~nm}$ films show that the $h c p$ phase grains are on average 10 and $11 \mathrm{~nm}$ in size, respectively. In addition, the $50 \mathrm{~nm}$-thick film consists of $9 \% h c p$ phase, whereas the $100 \mathrm{~nm}$ thick film contains $6 \% h c p$ phase. For comparison, the average grain size of the $f c c$ matrix phase was determined by measuring 178 grains from the corresponding reliability maps (Figs. $2 \mathrm{a}$ and $2 \mathrm{~b})$. The $f c c$ grains in the $50 \mathrm{~nm}$ film exhibit a narrower Gaussian distribution centered at approximately $23 \mathrm{~nm}$ (Fig. 2c), while those in the $100 \mathrm{~nm}$-thick film show a broader distribution centered at around $40 \mathrm{~nm}$ (Fig. 2d).

In summary, we demonstrate for the first time the morphology of nanoscale $h c p$ phase nickel that is present in as-deposited nanocrystalline PLD nickel films of different thicknesses.

This work was also supported by the Division of Materials Science and Engineering, Office of Basic Energy Sciences, U.S. Department of Energy. Sandia National Laboratories is a multiprogram laboratory operated by Sandia Corporation, a wholly owned subsidiary of Lockheed Martin company, for the U.S. Department of Energy's National Nuclear Security Administration under contract DE-AC04-94AL85000. 
References

[1] K. Hattar, 'Thermal and Mechanical Stability of nanocrystalline fcc metals", Ph. D. Dissertation, The University of Illinois - Urbana Champaign (2009)

[2] L. N. Brewer et al., Adv. Mater. 22 (2010) 1161

[3] K. Hattar et al., Acta Mater. 56 (2008) 794

[4] K. J. Ganesh et al., Microsc. Microanal. 16 (2010) 614

[5] E. F. Rauch et al., Microsc. Anal. Nanotech Suppl. November (2008) S5

FIG. 1. (a) and (b) Bright-field TEM image of the 50 and $100 \mathrm{~nm}$ films, (c) and (d) combined phase and reliability maps from the $50 \mathrm{~nm}$ film showing both $h c p$ and $f c c$ phase grains, and from the $100 \mathrm{~nm}$ film showing $h c p$ phase grains primarily at the $f c c$ phase grain boundaries.

\section{FIG. 2.}

(a) and (b) Reliability maps corresponding to FIG. 1 (c) and (d) for the films 50 and $100 \mathrm{~nm}$ thick, which are used to determine $f c c$ grain size in the two films, (c) and (d) $f c c$ grain size distribution in the 50 and $100 \mathrm{~nm}$ films.
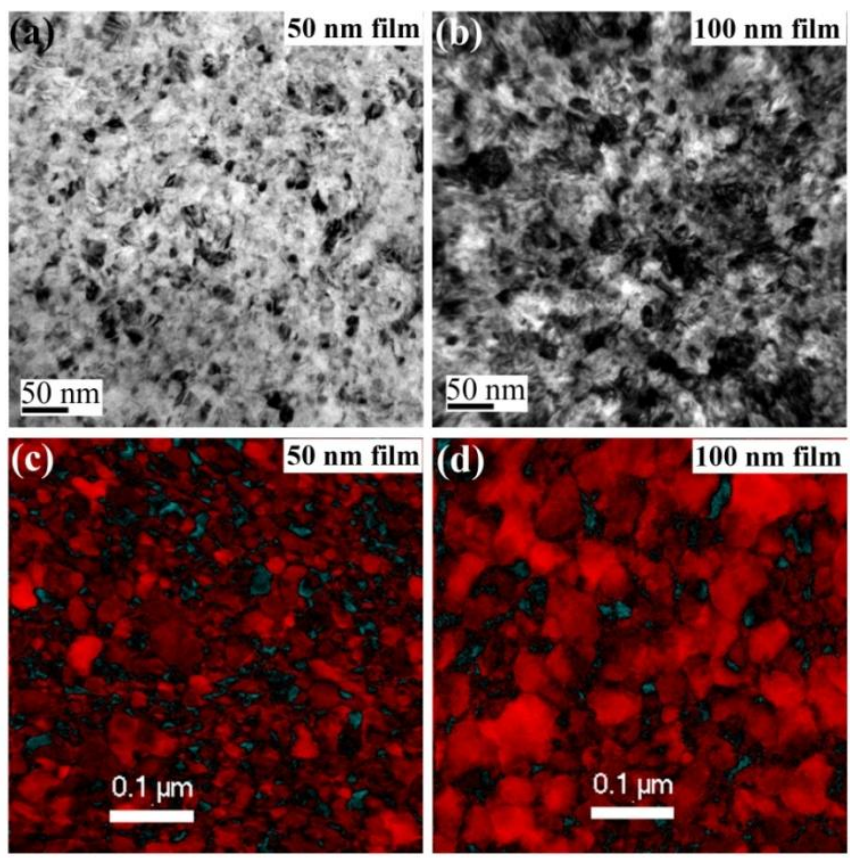

- $f c c$ phase $\quad$ hcp phase

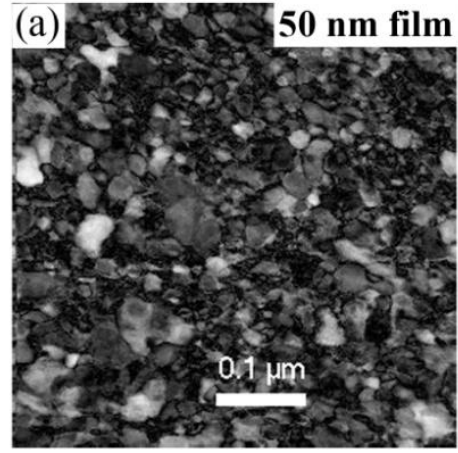

(c)

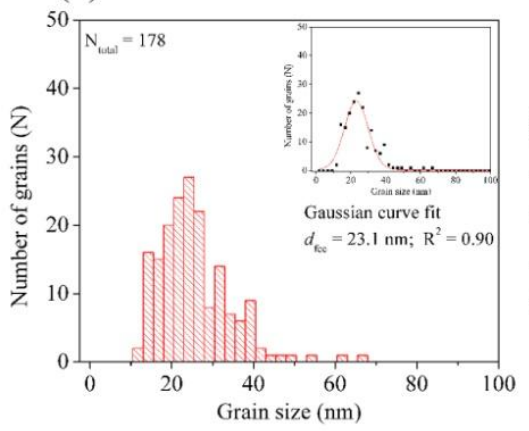

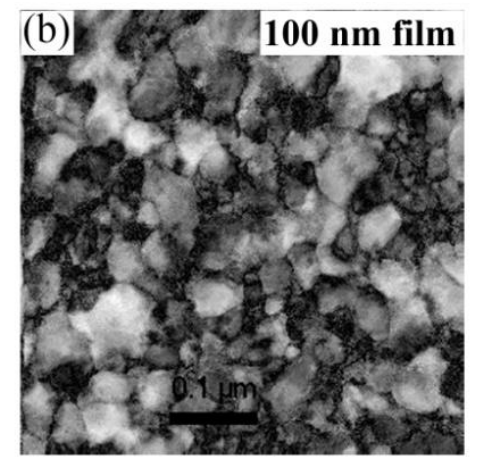

(d)

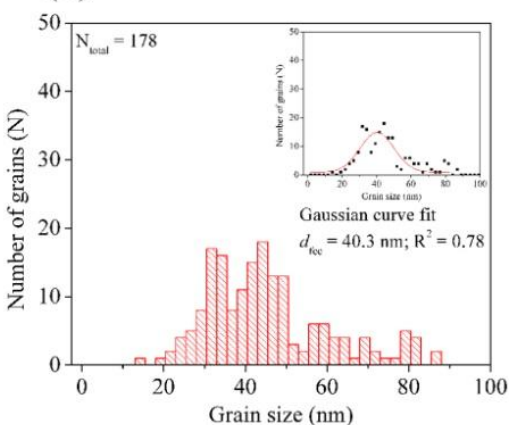

